\title{
On the theory of an adiabatic field dynamics in the Kerr medium with distributed gain and dispersion
}

\author{
Sergei K. TURITSYN ${ }^{1,2, *}$ \\ ${ }^{1}$ Aston Institute of Photonic Technologies, Aston University, Birmingham, B4 7ET, UK \\ ${ }^{2}$ Novosibirsk State University, Novosibirsk, 630090, Russia \\ *Corresponding author: s.k.turitsyn@aston.ac.uk
}

Compiled February 2, 2019

\begin{abstract}
A general theory is presented for the adiabatic field evolution in a nonlinear Kerr-medium with distributed amplification and varying dispersion. Analytical expression is derived linking parameter of the adiabaticity, gain distribution and dispersion profile. As a particular example, an optical pulse compressor based on the adiabatic dynamics is considered. (๑) 2019 Optical Society of America
\end{abstract}

\section{http://dx.doi.org/10.1364/ao.XX.XXXXXX}

Nonlinear optical systems offer a number of practical applications based on the nonlinear science concepts, ranging from solitons and supercontinuum to the nonlinear Fourier transform and parametric amplification. Interplay between dispersion and nonlinearity, combined with distributed amplification create a number of opportunities for experimental implementation of fascinating nonlinear dynamics. Fibre-optic is especially attractive due to an excellent waveguiding and relative simplification of the corresponding underlying models [1,2]. This Letter deals with the general mathematical theory of the adiabatic optical pulse evolution in the nonlinear Kerr medium with gain and dispersion, but without loss of generality, the fibre-optic notations will be used and specific pulse compression example will be examined in what follow.

There are several key methods of laser pulse compression using fibre optics. First is the so-called fibre grating compression approach that exploits normal dispersion fibre dynamics $[3,4]$. Propagation in the normal dispersion spectrally broadens pulses and produces via self-phase modulation effect a quasi-linear (which is exactly linear for parabolic pulses) temporal chirp. A the next stage the pulses are compressed up to the temporal widths determined by its spectral bandwidth using elements with anomalous dispersion.

Second approach is the so-called multi-soliton compression technique [5] that is based on higher-order nonlinear soliton dynamics in the anomalous dispersion. Evolution of the higherorder solitons along the fibre is characterised by periodic compression of the input state leading to narrow temporal spike. This nonlinear compression method allows for strong compression, but requires a careful adjustment of the input power to control the point of maximal compression. Another well-known challenge in this technique is the appearance of a broad pedestal that contains a large fraction of the total energy. Advantage of active fibre based compressors is in possibility to use low-power pulse sources and combination of compression and amplification [6-8]. Amplifying nonlinear effect compressors can enhance both fibre grating compression and multi-soliton method. For instance, in the amplifying fibre with normal dispersion the input pulse evolves into more clean linearly chirped parabolic pulse, which can be efficiently compressed [7].

A possibility to use varying dispersion [9] in fibre provides additional possibility for pulse compression. One of the attractive and robust compression schemes is adiabatic soliton compression in the dispersion decreasing fibre, which ensures time-bandwidth limited output [10-16]. Tapering can be applied to gas-filled hollow-core anti-resonant fibers to achieve generation of extreme ultraviolet dispersive waves [17]. Strict mathematical conditions for adiabatic soliton dynamics have been presented for an axially nonuniform (tapered) fibre in [10]. Most of fibre-based dispersion-tailored compressors use tapered fibre spans with varying diameter that changes simultaneously dispersion, effective nonlinear coefficient and effective refraction index. Adiabatic compression technique is based on the perturbation theory of the nonlinear Schrödinger equation that ensures smooth soliton evolution that preserves some integral pulse characteristics. Mathematically, the adiabatic condition is that the product of the effective gain (or loss) coefficient and the soliton period is less than unity. This condition will be discussed in detail below. True adiabatic compression does not produce any extra chirp maintaining transform limited pulses.

In this Letter mathematical theory of the adiabatic pulse compression in nonlinear Kerr medium with both varying dispersion and distributed amplification will be examined. Though our analysis has some similarity with the tapered fibre $[10,11]$ we consider different and more generic design that can be applied beyond the tapered waveguide systems. Note that this general consideration does not include effects such as, e.g. higher-order dispersion, Raman scattering and other effects that limit applications of the approach. Typically these limiting effects vary from one application to another and are less general than the considered generic system. Therefore, we leave analysis of the limiting impact of the higher order terms beyond the scope of this Letter. It is interesting to point out that in some situations detrimental effect of Raman scattering and higher-order dispersion can interfere compensating each other and keeping the 
pulse compression adiabatic [18]

\section{A. Basics of an adiabatic compressor}

Optical field $E(z, t)$ propagation down the amplifying optical medium with Kerr nonlinearity and varying group velocity dispersion is governed by the generalised nonlinear Schrödinger equation (NLSE) with loss and gain (see for details, e.g. $[1,2,19])$.

$$
i \frac{\partial E}{\partial z}-\frac{\beta_{2}(z)}{2} \frac{\partial^{2} E}{\partial t^{2}}+\gamma|E|^{2} E=-i \alpha E+i g(z) E .
$$

Here $\beta_{2}(z)=-\left|\beta_{2}(0)\right| s^{2}(z)$ is the group velocity dispersion, dimensionless function $s^{2}(z)$ (normalised with the condition $s(0)=1$ ) defines variations of dispersion profile along the waveguide, we consider here only anomalous dispersion (in fibre-optic terms) with $\beta_{2}<0, \gamma$ is the nonlinear Kerr coefficient, $\alpha$ is linear loss, and $g(z)$ describes a distributed gain. Consider waveforms (e.g. pulses) with the characteristic temporal duration $T_{0}$. It is assumed here that the gain distribution along the waveguide is accustomed and our goal is to determine the longitudinal dispersion profile that provides an effective adiabatic propagation of optical pulse with a given power level.

Let us transform the Eq. 1 using the following substitution and a straightforward change of variables:

$$
E(z, t)=\sqrt{\frac{\left|\beta_{2}(0)\right|}{\gamma T_{0}^{2}}} \times s(z) \times q(Z, T)
$$

here

$$
Z=\frac{\int_{0}^{z} s^{2}\left(z^{\prime}\right) d z^{\prime}}{L_{d i s}}, \quad L_{d i s}=\frac{T_{0}^{2}}{\left|\beta_{2}(0)\right|}, \quad T=\frac{t}{T_{0}} .
$$

Consider a general design of the dispersion profile varying with $z$ that can provide for the adiabatic evolution with the targeted adiabaticity parameter $\epsilon \ll 1$ for any given gain distribution $g(z)$. It is easy to check, that by selecting a normalised dispersion $s(z)$ that satisfies the following equation:

$$
\frac{d s}{d z}=-\frac{\epsilon}{L_{d i s}} s^{3}+[g(z)-\alpha] s,
$$

we can ensure that the dynamics of the field $q(Z, T)$ is governed by the following master equation that presents classical nonlinear Schrödinger equation (NLSE) with the linear perturbation:

$$
i \frac{\partial q}{\partial Z}+\frac{1}{2} \frac{\partial^{2} q}{\partial T^{2}}+|q|^{2} q=i \epsilon q
$$

Assuming $\epsilon$ to be small, this equation describes adiabatic evolution of the field $q(Z, T)$ with $Z$. This is a master model for waveguide systems based on the adiabatic signal dynamics. One of the advantages of the adiabatic approach is a possibility to use a powerful mathematical tool for the system design. The nonlinear Schrödinger equation (that is Eq. 5 with $\epsilon=0$ ) is integrable by the so-called inverse scattering transform (IST) method [20] (also known as the nonlinear Fourier transform, see e.g. recent review of its applications in optical communications [21]).

In the case of small $|\epsilon| \ll 1$ optical field evolution can be analysed using the perturbation theory based on the IST $[19,22]$. Recall a well-known fact that for a single soliton having initial shape $q(T, Z=0)=\lambda_{0} / \cosh \left[\lambda_{0} T\right]$, the adiabatic evolution will be given by (see e.g. $[2,19])$ :

$$
q(T, Z)=\frac{\lambda(Z) \times \exp [i \sigma(Z)]}{\cosh [\lambda(Z) T]}
$$

where evolution of a soliton amplitude is given by $\lambda(z)=$ $\lambda_{0} \exp [2 \epsilon Z]$ and phase by $\sigma(Z)=\lambda_{0}^{2} \times(1-\exp [4 \epsilon Z]) /(8 \epsilon)$. In dimension units an input pulse power is determined as $P_{\text {in }}=\lambda_{0}^{2}\left|\beta_{2}(0)\right| /\left(\gamma T_{0}^{2}\right)$.

Evolution of a pulse full-width-at-half-maximum $T_{F W H M}$ and corresponding broadening or compression is given by $T_{\text {FWHM }}(Z)=1.763 \exp [-2 \epsilon Z] / \lambda_{0}$. It is seen that positive $\epsilon$ (effective gain) leads to temporal pulse compression, while negative $\epsilon$ (effective loss) corresponds to temporal broadening and spectral compression. This is the basics of the adiabatic compression of soliton pulse in the media described by Eq. 5 . One can see that parameter $\epsilon$ defines rate of the adiabatic pulse compression. It can be controlled by the appropriate design of the dispersion profile for a given distributed gain $g(z)$. It is important to point out that adiabatic evolution is not linked to single soliton dynamics and might be exploited for various inputs waveforms.

\section{B. Dispersion profile for adiabatic dynamics}

A general solution of the equation 4 for the normalised dispersion profile $s(z)$ with the initial condition $s(0)=1$ is found as:

$$
s^{2}(z)=\frac{\exp [F(z)]}{1+\frac{2 \epsilon}{L_{d i s}} \int_{0}^{z} \exp \left[F\left(z^{\prime}\right) d z^{\prime}\right]},
$$

here

$$
F(z)=2 \int_{0}^{z}\left(g\left(z^{\prime}\right)-\alpha\right) d z^{\prime}
$$

The Eqs. 7-8 present the main result of the Letter. It gives profile of the longitudinal dispersion variation $s(z)$ that for a given gain distribution allows to achieve strictly adiabatic regime governed by Eq. 5. This expression generalizes result for dispersion profile tailored to the gain to achieve lossless NLSE propagation [23]. In the case of the lossless $\operatorname{NLSE}(F(z)=0)$, Eq. 7 reproduces a well-known result $s^{2}(z)=1 /\left(1+2 \epsilon z / L_{\text {dis }}\right)$. Note that though $\epsilon$ is small, the second term in the denominator is not necessarily also small. Using Eqs. 7-8 we can explicitly express the variable $\mathrm{Z}$ as

$$
Z=\frac{\int_{0}^{z} s^{2}\left(z^{\prime}\right) d z^{\prime}}{L_{d i s}}=\frac{1}{\epsilon} \ln \left[1+\frac{2 \epsilon}{L_{d i s}} \int_{0}^{z} \exp \left[F\left(z^{\prime}\right) d z^{\prime}\right]\right] .
$$

Note that distributed gain can be implemented in various ways. Below several particular examples of the application of a general theory are examined.

\section{Constant adiabatic gain or loss}

Now consider several specific examples of the compressor design. Relatively short spans of active waveguide can provide with a good accuracy a constant (uniform along the waveguide length) gain $g_{0}$. In the case of a constant loss or gain (e.g. in active waveguide) $g(z)-\alpha=g_{0}$, the expression for dispersion profile $s(z)$ is simplified.

$$
s^{2}(z)=\frac{\exp \left[2 g_{0} z\right]}{1+\epsilon\left(\exp \left[2 g_{0} z\right]-1\right) /\left(g_{0} L_{d i s}\right)},
$$

For $\epsilon=0$ from here we recover the well-known result by Tajima [9]: $s^{2}=\exp \left[2 g_{0} z\right]$. In the dimension units the optical field reads:

$$
E(z, t)=\sqrt{\frac{\left|\beta_{2}(0)\right|}{\gamma T_{0}^{2}}} \times \frac{\exp \left[g_{0} z\right] \times q\left(Z, \frac{t}{T_{0}}\right)}{\left(1+\epsilon\left[\exp \left(2 g_{0} z\right)-1\right] /\left(g_{0} L_{d i s}\right)\right)^{1 / 2}},
$$




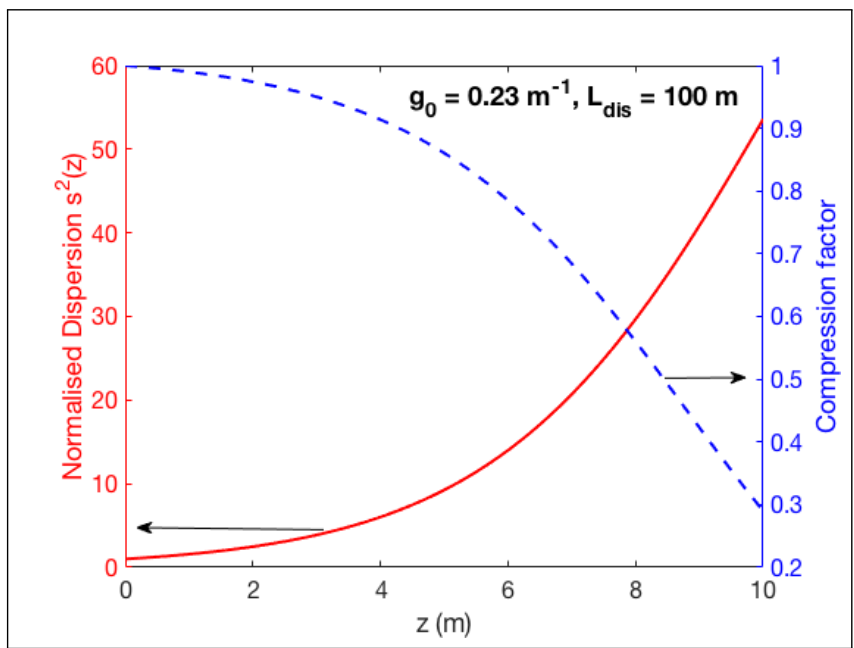

Fig. 1. Normalised dispersion $s^{2}(z)$ for $\epsilon=0.2, g_{0}=0.23 \mathrm{~m}^{-1}$ ( $2 \mathrm{~dB} / \mathrm{m}), L_{d i s}=100 \mathrm{~m}$ and compression factor of the pulse width at half maximum $T_{F W H M}(z) / T_{F W H M}(0)=1 /(1+$ $\left.\epsilon\left[\exp \left(2 g_{0} z\right)-1\right] /\left(g_{0} L_{\text {dis }}\right)\right)^{2}$ with distance

where $\epsilon Z=\ln \left[1+\epsilon\left(\exp \left[2 g_{0} z\right]-1\right) /\left(g_{0} L_{\text {dis }}\right)\right]$. For a soliton pulse power evolution down the waveguide is given by:

$$
|E(z, t)|^{2}=\frac{P_{\mathcal{S}}(z)}{\cosh ^{2}\left[\frac{t}{\tau(z)}\right]},
$$

with varying pulse width parameter $\tau(z)$

$$
\tau(z)=\frac{T_{0}}{\lambda_{0}\left[1+\epsilon\left[\exp \left(2 g_{0} z\right)-1\right] /\left(g_{0} L_{\text {dis }}\right)\right]^{2}}
$$

and power

$$
P_{S}(z)=\frac{\lambda_{0}^{2}\left|\beta_{2}(0)\right|}{\gamma T_{0}^{2}} \times \exp \left(2 g_{0} z\right)\left(1+\frac{\epsilon\left[\exp \left(2 g_{0} z\right)-1\right]}{g_{0} L_{\text {dis }}}\right)^{3} .
$$

It is seen from these relations that the considered adiabatic regime is more efficient with lower $L_{\text {dis }}$ (e.g. higher anomalous dispersion). Note the exponential growth factor in Eq. 10 that dominates the first part of the $s^{2}(z)$ change with $z$. The term with $\epsilon$ that defines adiabatic evolution contributes at the point where gain makes term $\epsilon\left[\exp \left(2 g_{0} z\right)-1\right] /\left(g_{0} L_{\text {dis }}\right)$ comparable with unity. It is seen that the adiabatic compression either requires high variation of dispersion (in case of not small $L_{d i s}$, or small $L_{d i s}$ corresponding to short pulse and/or high level of dispersion. Once more, we would like to stress that we do not aim here to present any particular implementation, but rather a mathematical theory for a family of devices that can use different platforms. Figure 1 depicts normalised dispersion profile $s^{2}(z)$ for $\epsilon=0.2, g_{0}=0.23 \mathrm{~m}^{-1}$ $(2 \mathrm{~dB} / \mathrm{m})$ and $L_{d i s}=100 \mathrm{~m}$. The level of compression with propagation is also shown for the pulse width at half maximum $T_{F W H M}(z) / T_{F W H M}(0)=1 /\left(1+\epsilon\left[\exp \left(2 g_{0} z\right)-1\right] /\left(g_{0} L_{\text {dis }}\right)\right)^{2}$. It is seen that smaller gain allows to keep dispersion variation within smaller range. Figure 2 presents similar plots, but for $g_{0}=0.115 \mathrm{~m}^{-1}(1 \mathrm{~dB} / \mathrm{m})$ and $L_{\text {dis }}=30 \mathrm{~m}$ with all other parameters the same.

\section{Distributed Raman amplification}

In the case of the often used backward Raman amplification, for unsaturated gain a function $g(z)$ is given by: $g(z)=$

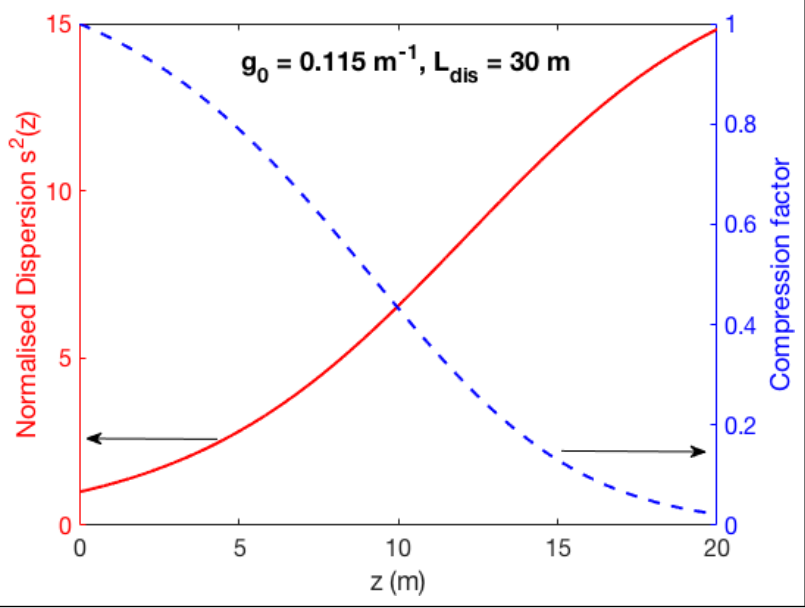

Fig. 2. Normalised dispersion $s^{2}(z)$ for $\epsilon=0.2, g_{0}=$ $0.115 \mathrm{~m}^{-1}(1 \mathrm{~dB} / \mathrm{m}), L_{d i s}=30 \mathrm{~m}$ and compression factor of the pulse width at half maximum $T_{F W H M}(z) / T_{F W H M}(0)=$ $1 /\left(1+\epsilon\left[\exp \left(2 g_{0} z\right)-1\right] /\left(g_{0} L_{\text {dis }}\right)\right)^{2}$ with distance

$0.5 g_{R} P_{p b} \exp \left[-2 \alpha_{p}(L-z)\right]$, where $g_{R}$ is the Raman gain coefficient, $P_{p b}$ is the backward pump power injected at $z=L$, and $\alpha_{p}$ is the loss at the pump wavelength. In this case,

$$
F_{b}(z)=\frac{g_{R} P_{p b} \exp \left(-2 \alpha_{p} L\right)}{2 \alpha_{p}} \times\left[\exp \left(2 \alpha_{p} z\right)-1\right]-2 \alpha z .
$$

We would like to stress that in the considered scheme Raman gain itself is not required to be adiabatic (small), but it is a combination of the distributed gain and varying dispersion that leads to the adiabatic dynamics.

Figure 3 shows a normalised dispersion profile $s^{2}(z)$ computed for the following parameters for the SMF-28 $\alpha=$ $0.023 \mathrm{~km}^{-1}$ at $1550 \mathrm{~nm}, g_{R}=0.4(W \times \mathrm{km})^{-1}, \alpha_{p}=$ $0.0285 \mathrm{~km}^{-1}$, several values of $P_{p b}=1,3,5 \mathrm{~W}, \epsilon=0.2$ and $L_{\text {dis }}=5 \mathrm{~km}$.

In the similar manner one we can derive expressions for the cases of forward and combined forward and backward distributed Raman amplification schemes. Unsaturated forward pumped Raman amplification yields:

$$
F_{f}(z)=\frac{g_{R} P_{p f}}{2 \alpha_{p}} \times\left[1-\exp \left(-2 \alpha_{p} z\right)\right]-2 \alpha z .
$$

A combined forward and backward pumping scheme gives:

$F_{b f}(z)=\frac{g_{R} P_{p f}\left(1-e^{-2 \alpha_{p} z}\right)+g_{R} P_{p b}\left(e^{2 \alpha_{p}(z-L)}-e^{-2 \alpha_{p} L}\right)}{2 \alpha_{p}}-2 \alpha z$.

We would like to stress that distributed Raman amplification alone can be exploited for adiabatic compression. However, in this case condition of adiabaticity imposes severe limitations of the level of amplification. Adjusting dispersion variation profile to the chosen amplification scheme allows one to relax these restrictions.

\section{E. Axially non-uniform fibre}

Let us recall, for completeness and comparison, a theory of adiabatic pulse soliton compression in the dispersion-decreasing 


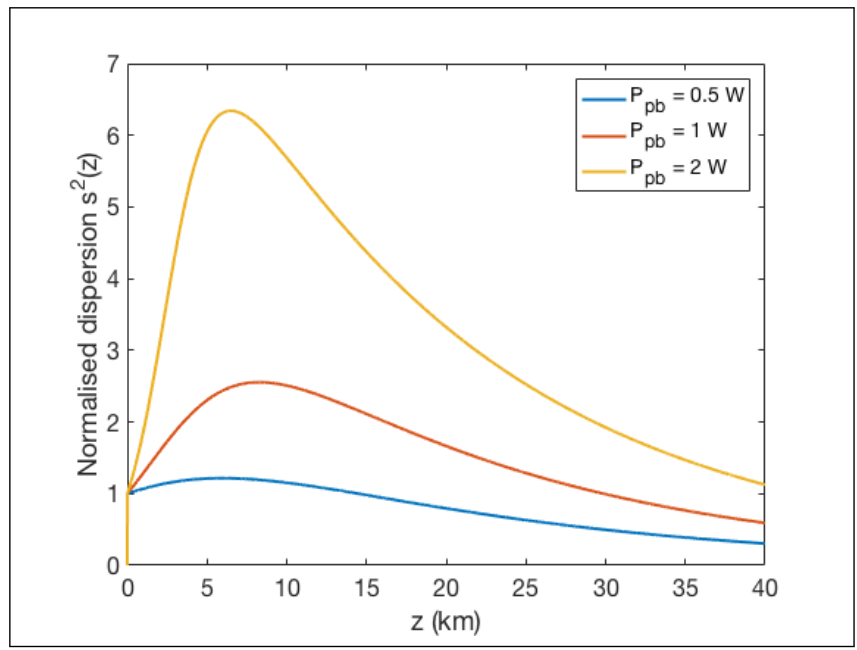

Fig. 3. A normalised dispersion profile $s^{2}(z)$, here $\alpha=$ $0.023 \mathrm{~km}^{-1}$ at $1550 \mathrm{~nm}, g_{R}=0.4(W \times \mathrm{km})^{-1}, \alpha_{p}=$ $0.0285 \mathrm{~km}^{-1}$, backward pump powers are $P_{p b}=0.5,1,2 \mathrm{~W}$, $\epsilon=0.2$ and $L_{\text {dis }}=5 \mathrm{~km}$.

tapered fibre. The equation governing propagation of the envelope $U(z, t)$ of light wave in non-uniform fibre reads (see for details of notations and definitions in $[10,11])$ :

$$
i \frac{\partial U}{\partial z}-\frac{k_{2}(z)}{2} \frac{\partial^{2} U}{\partial t^{2}}+\gamma(z)|U|^{2} U=i(\tilde{g}(z)-\alpha) U-\frac{i}{2 f} \frac{d f}{d z} U .
$$

Here $k_{2}(z)$ is a varying group velocity dispersion, $f(z)$ corresponds to variation of the effective refractive index and $\gamma(z)$ describes gradual change of the effective mode area along the fibre [10,11, 15]. The term with $f(z)$ ensures the conservation of the total photon number in non-uniform optical fibre in the absence of signal attenuation. For the sake of clarity, we do not include here the mean frequency variation effect that can be added in a straightforward way following detailed description in [11]. Applying similar transform:

$$
U(z, t)=\sqrt{\frac{\left|k_{2}(0)\right|}{\gamma(z) T_{0}^{2}}} \times s(z) \times w(\eta, T)
$$

where

$$
\eta=\frac{\int_{0}^{z} s^{2}\left(z^{\prime}\right) d z^{\prime}}{L_{d i s}}, \quad L_{d i s}=\frac{T_{0}^{2}}{\left|k_{2}(0)\right|}, \quad T=\frac{t}{T_{0}} .
$$

one can derive adiabatically perturbed NLSE

$$
i \frac{\partial w}{\partial \eta}+\frac{1}{2} \frac{\partial^{2} w}{\partial T^{2}}+|w|^{2} w=i \epsilon w
$$

in this case the expression for dispersion profile $s(z)$ reads

$$
\frac{d s}{d z}=-\frac{\epsilon}{L_{d i s}} s^{3}+\left[g(z)-\alpha-\frac{1}{2 f} \frac{d f}{d z}+\frac{1}{2 \gamma} \frac{d \gamma}{d z}\right] s,
$$

The analytical solution given by Eqs. 7-8 can be also used in this case. Distributed amplification can be exploited to provide additional degrees of freedom for design of such non-uniform fibre-based adiabatic compression systems.

In conclusion, a mathematical theory is presented that links optical systems with Kerr nonlinearity, distributed amplification and varying dispersion to integrable model with strictly adiabatic field evolution. Though focus of this Letter is on an optical pulse compression, the theory is not limited to soliton dynamics, compression and monotonic change of dispersion. It can also be used for applications with periodic changes of dispersion and gain. Analytical expression is derived for the dispersion profile that links parameter of adiabaticity (related to the compression rate), gain distribution and input pulse parameters. It should be stressed that the considered theory is limited by various higher-order effects that should be taken into account when pulse becomes narrow. Consideration of the higher-order effects is straightforward [11, 18], however, it is beyond the scope of this Letter.

This work was supported by the Russian Science Foundation (Grant No. 17-72-30006). I would like to thank Elena G. Turitsyna for assistance in the preparation of figures and Auro Perego for discussions.

\section{REFERENCES}

1. G. P. Agrawal, The Nonlinear Fiber Optics (Academic press, 2007), 4th ed.

2. L. F. Mollenauer and J. Gordon, Solitons in optical fiber (Academic Press, 2006).

3. C. V. Shank, R. L. Fork, R. Yen, R. H. Stolen, and W. J. Tomlinson, Appl. Phys. Lett. 40, 761 (1982).

4. R. Fork, C. B. Cruz, P. Becker, and C. Shank, Opt. Lett. 12(7), 483 (1987).

5. L. F. Mollenauer, R. H. Stolen, J. P. Gordon, and W. J. Tomlinson, Opt. Lett. 8, 289 (1983).

6. K. Smith and L. F. Mollenauer, Opt. Lett. 14, 751 (1989).

7. K. Tamura and M. Nakazawa, Opt. Lett. 21, 68 (1996).

8. M. L. Quiroga-Teixeiro, D. Anderson, P. Andrekson, A. Berntson, and M. Lisak, JOSA B 13, 687 (1996).

9. K. Tajima, Opt. Lett. 12, 54 (1987).

10. H. H. Kuehl, J. Opt. Soc. Am. B 5, 709 (1988).

11. S. V. Chernikov and P. V. Mamyshev, J. Opt. Soc. Am. B 8, 1633 (1991).

12. V. A. Bogatyrev, M. M. Bubnov, E. M. Dianov, A. S. Kurkov, P. V. Mamyshev, A. M. Prokhorov, S. D. Rumyantsev, V. A. Semenov, S. L. Semenov, A. A. Sysoliatin, S. V. Chernikov, A. N. Gur'yanov, G. G. Devyatykh, and S. I. Miroshnichenko, J. Light. Technol. 9, 561 (1991).

13. S. V. Chernikov, E. M. Dianov, D. J. Richardson, and D. N. Payne, Opt. Lett. 18, 476 (1993).

14. E. M. Dianov, P. V. Mamyshev, A. M. Prokhorov, and S. V. Chernikov, Opt. Lett. 14, 1008 (1989).

15. J. C. Travers, J. M. Stone, A. B. Rulkov, B. A. Cumberland, A. K. George, S. V. Popov, J. C. Knight, and J. R. Taylor, Opt. Express 15, 13203 (2007).

16. M. A. Foster, A. L. Gaeta, Q. Cao, and R. Trebino, Opt. Express 13, 6848 (2005).

17. M. S. Habib, C. Markos, J. E. Antonio-Lopez, R. A. Correa, O. Bang, and M. Bache, Opt. Express 26, 24357 (2018).

18. P. V. Mamyshev, P. G. J. Wigley, J. Wilson, G. I. Stegeman, V. A. Semeonov, E. M. Dianov, and S. I. Miroshnichenko, Phys. Rev. Lett. 71, 73 (1993).

19. A. Hasegawa and Y. Kodama, Solitons in optical communications (Oxford University Press, 1995).

20. V. E. Zakharov and A. B. Shabat, Sov. Phys. JETP 34, 62 (1972).

21. S. K. Turitsyn, J. E. Prilepsky, S. T. Le, S. Wahls, M. K. L. L. Frumin, and S. A. Derevyanko, Optica 4, 307 (2017).

22. Y. S. Kivshar and B. A. Malomed, Rev. Mod. Phys. 61, 763 (1989).

23. G. H. M. van Tartwijk, R.-J. Essiambre, and G. P. Agrawal, Opt. Lett. 21, 1978 (1996). 


\section{FULL REFERENCES}

1. G. P. Agrawal, The Nonlinear Fiber Optics (Academic press, 2007), 4th ed.

2. L. F. Mollenauer and J. Gordon, Solitons in optical fiber (Academic Press, 2006).

3. C. V. Shank, R. L. Fork, R. Yen, R. H. Stolen, and W. J. Tomlinson, "Compression of femtosecond optical pulses," Appl. Phys. Lett. 40, 761-763 (1982).

4. R. Fork, C. B. Cruz, P. Becker, and C. Shank, "Compression of optical pulses to six femtoseconds by using cubic phase compensation," Opt. Lett. 12(7), 483 (1987).

5. L. F. Mollenauer, R. H. Stolen, J. P. Gordon, and W. J. Tomlinson, "Extreme picosecond pulse narrowing by means of soliton effect in single-mode optical fibers," Opt. Lett. 8, 289-291 (1983).

6. K. Smith and L. F. Mollenauer, "Experimental observation of adiabatic compression and expansion of soliton pulses over long fiber paths," Opt. Lett. 14, 751-753 (1989).

7. K. Tamura and M. Nakazawa, "Pulse compression by nonlinear pulse evolution with reduced optical wave breaking in erbium-doped fiber amplifiers," Opt. Lett. 21, 68-70 (1996).

8. M. L. Quiroga-Teixeiro, D. Anderson, P. Andrekson, A. Berntson, and M. Lisak, "Efficient soliton compression by fast adiabatic amplification," JOSA B 13, 687-692 (1996).

9. K. Tajima, "Compensation of soliton broadening in nonlinear optical fibers with loss," Opt. Lett. 12, 54-56 (1987).

10. H. H. Kuehl, "Solitons on an axially nonuniform optical fiber," J. Opt. Soc. Am. B 5, 709-713 (1988).

11. S. V. Chernikov and P. V. Mamyshev, "Femtosecond soliton propagation in fibers with slowly decreasing dispersion," J. Opt. Soc. Am. B 8, 16331641 (1991).

12. V. A. Bogatyrev, M. M. Bubnov, E. M. Dianov, A. S. Kurkov, P. V. Mamyshev, A. M. Prokhorov, S. D. Rumyantsev, V. A. Semenov, S. L. Semenov, A. A. Sysoliatin, S. V. Chernikov, A. N. Gur'yanov, G. G. Devyatykh, and S. I. Miroshnichenko, "A single-mode fiber with chromatic dispersion varying along the length," J. Light. Technol. 9, 561-566 (1991).

13. S. V. Chernikov, E. M. Dianov, D. J. Richardson, and D. N. Payne, "Soliton pulse compression in dispersion-decreasing fiber," Opt. Lett. 18, 476-478 (1993).

14. E. M. Dianov, P. V. Mamyshev, A. M. Prokhorov, and S. V. Chernikov, "Generation of a train of fundamental solitons at a high repetition rate in optical fibers," Opt. Lett. 14, 1008-1010 (1989).

15. J. C. Travers, J. M. Stone, A. B. Rulkov, B. A. Cumberland, A. K. George, S. V. Popov, J. C. Knight, and J. R. Taylor, "Optical pulse compression in dispersion decreasing photonic crystal fiber," Opt. Express 15, 1320313211 (2007).

16. M. A. Foster, A. L. Gaeta, Q. Cao, and R. Trebino, "Soliton-effect compression of supercontinuum to few-cycle durations in photonic nanowires," Opt. Express 13, 6848-6855 (2005).

17. M. S. Habib, C. Markos, J. E. Antonio-Lopez, R. A. Correa, O. Bang, and $\mathrm{M}$. Bache, "Multi-stage generation of extreme ultraviolet dispersive waves by tapering gas-filled hollow-core anti-resonant fibers," Opt. Express 26, 24357-24371 (2018).

18. P. V. Mamyshev, P. G. J. Wigley, J. Wilson, G. I. Stegeman, V. A. Semeonov, E. M. Dianov, and S. I. Miroshnichenko, "Adiabatic compression of schrödinger solitons due to the combined perturbations of higherorder dispersion and delayed nonlinear response," Phys. Rev. Lett. 71, 73-76 (1993).

19. A. Hasegawa and Y. Kodama, Solitons in optical communications (Oxford University Press, 1995).

20. V. E. Zakharov and A. B. Shabat, "Exact theory of two-dimensional selffocusing and one-dimensional self-modulation of waves in nonlinear media," Sov. Phys. JETP 34, 62 (1972).

21. S. K. Turitsyn, J. E. Prilepsky, S. T. Le, S. Wahls, M. K. L. L. Frumin, and S. A. Derevyanko, "Nonlinear fourier transform for optical data processing and transmission: advances and perspectives," Optica 4, 307-322 (2017).

22. Y. S. Kivshar and B. A. Malomed, "Dynamics of solitons in nearly integrable systems," Rev. Mod. Phys. 61, 763-915 (1989)

23. G. H. M. van Tartwijk, R.-J. Essiambre, and G. P. Agrawal, "Dispersiontailored active-fiber solitons," Opt. Lett. 21, 1978-1980 (1996). 